\title{
Review of the emerging role of optical polarimetry in characterization of pathological myocardium
}

\author{
Iftikhar Ahmad
}




\title{
Review of the emerging role of optical polarimetry in characterization of pathological myocardium
}

\author{
Iftikhar Ahmad* \\ Center for Nuclear Medicine and Radiotherapy (CENAR), Quetta, Pakistan
}

\begin{abstract}
Myocardial infarction (MI), a cause of significant morbidity and mortality, is typically followed by microstructural alterations where the necrotic myocardium is steadily replaced with a collagen scar. Engineered remodeling of the fibrotic scar via stem cell regeneration has been shown to improve/restore the myocardium function after MI. Nevertheless, the heterogeneous nature of the scar patch may impair the myocardial electrical integrity, leading to the formation of arrhythmogenesis. Radiofrequency ablation (RFA) offers an effective treatment for focal arrhythmias where local heating generated via electric current at specific spots in the myocardium ablate the arrhythmogenic foci. Characterization of these myocardial pathologies (i.e., infarcted, stem cell regenerated, and RFA-ablated myocardial tissues) is of potential clinical importance. Optical polarimetry, the use of light to map and characterize the polarization signatures of a sample, has emerged as a powerful imaging tool for structural characterization of myocardial tissues, exploiting the underlying highly fibrous tissue nature. This study aims to review the recent progress in optical polarimetry pertaining to the characterization of myocardial pathologies while describing the underlying biological rationales that give rise to the optical imaging contrast in various pathologies of the myocardium. Future possibilities of and challenges to optical polarimetry in cardiac imaging clinics are also discussed. @ 2017 Society of Photo-Optical Instrumentation Engineers (SPIE) [DOI: 10.1117/1.JBO.22.10.100901]
\end{abstract}

Keywords: optical polarimetry; myocardial infarction; tissue anisotropy; stem cell therapy; radiofrequency ablation.

Paper 170260VR received May 2, 2017; accepted for publication Oct. 4, 2017; published online Oct. 27, 2017.

\section{Introduction}

Myocardial infarction (MI), a cause of significant morbidity and mortality, is typically followed by structural remodeling where the necrotic myocardium is steadily replaced with the deposition of collagen matrix, ultimately composing a heterogeneously distributed fibrotic scar at the infarct site, ${ }^{1,2}$ which may also facilitate a transition from healing to adverse effects such as aneurysmal deformation ${ }^{3,4}$ and the triggering and maintenance of electrical instability ${ }^{5-7}$ to form arrhythmogenic circuit. Engineered remodeling of the fibrotic scar patch via stem cell regenerative therapy may likely contribute to improve the structural integrity and subsequently the contractile function of the myocardium after MI; indeed, such regenerative therapy has been actively pursued with encouraging results. ${ }^{89}$ On the other hand, focal arrhythmias can be effectively treated with radiofrequency ablation (RFA), ${ }^{10}$ where local heating generated via electric current at specific spots in the myocardium ablate arrhythmogenic foci. The ablated lesion typically comprises a coagulative necrotic core surrounded by a rim region of intermixed viable and nonviable cells. ${ }^{11,12}$ For completeness, the orientation of myofibers in cardiac muscle-normal, infarcted, or regenerated-is crucial toward the conduction of electric potential and, thereby, heart contractile function.

Appropriate assessment of the aforementioned cardiac pathological states (i.e., infarcted, stem cell regenerated, and RFAablated) is of potential clinical importance. To this end, a variety of the prevailing medical imaging techniques such as ultrasound (US), magnetic resonance (MR), and computed tomography

*Address all correspondence to: Iftikhar Ahmad, E-mail: iahmadmp @ gmail.com
(CT) have been explored; however, each of these imaging modalities has its own advantages and limitations, as discussed elsewhere. ${ }^{13,14}$ Alternatively optical imaging, including optical polarimetry, represents a powerful tool in the investigation of structural characteristics of myocardial tissues, particularly those bearing structural remodeling such as infarction, stem cell regeneration, and RFA ablation. In particular, cardiac tissue comprises fibrous constituents (e.g., cardiomyocytes and collagen fibers), which are highly anisotropic in nature, a feature of prime significance for optical polarimetry evaluation of cardiac pathology. The structural anisotropy is closely related to the linear retardance and subsequently to optical birefringence, which is governed by the different refractive indices as experienced by light traveling in different spatial directions (e.g., along the length of the fibers versus perpendicular to the fibers). In this context, optical polarimetry has the potential to sensitively measure the linear retardance (both magnitude and direction) of the given cardiac tissue, generating a quantitative map of linear retardance across the analyzed tissue; any anomaly observed here would be of particular interest because it indicates crucial information about the underlying structural alterations. More specifically, the healthy zones of cardiac tissue are primarily comprised of well-organized aligned arrays of cardiomyocytes and would ensure a distribution of higher values of linear birefringence, compared to the pathological regions where significant disruption of the ordered striated architecture will result in a corresponding loss of the tissue anisotropy. Consequently, the noninvasive assessment of such optical signatures has the potential to shed light to the ultrastructural modeling of cardiac tissue.

\footnotetext{
$1083-3668 / 2017 / \$ 25.00$ @ 2017 SPIE
} 
It is worth mentioning that such applications of polarized light are not limited to cardiac tissues, ${ }^{13-17}$ rather have been described in the investigation of several other tissue types with various pathology platforms; indicative examples include skin and colon cancers, ${ }^{18,19}$ structural disorders of bladder wall, ${ }^{20}$ etc.

Recently, many studies have investigated polarized light measurements for the characterization of various pathologies pertaining to cardiac tissue, proving the growing interest of the research community for such applications. For instance, myocardial tissues with induced infarction and stem cell regenerative treatment have been assessed with optical polarimetry. ${ }^{16,17,21}$ Moreover, optical polarimetry has successfully demonstrated visualizing a typical myocardial RFA lesion. ${ }^{14}$ This later study has been recently extended to automatic, objective segmentation of the polarization image into three clinically relevant zones, including the healthy, the necrotic core, and the rim regions. ${ }^{13}$ In addition, the orientation of cardiomyocytes in cardiac muscle has previously been explored accurately with optical polarimetry. ${ }^{15,22}$ Collectively, these studies illustrate that the optical polarimetry holds much promise for the characterization of myocardial pathologies, including sketching of myofiber organization. In this context, herein, the status of optical polarimetry to visualize, identify, and quantify myocardial pathologies and speculate on its future clinical translation has been summarized. Specifically, I start with a brief introduction to the tissue optical polarimetry (i.e., Mueller matrix methodology), followed by illustrative applications in the assessment of myocardial pathologies and myofiber orientation. Finally, the possibilities and challenges to the future clinical translation are briefly discussed.

\section{Experimental Setup of Optical Polarization Imaging}

The complete description of the polarization state of light is given by Stokes vector, a four-element vector, represented as $\mathbf{S}=\left[\begin{array}{llll}\mathrm{I} & \mathrm{Q} & \mathrm{U} & \mathrm{V}\end{array}\right]^{T}$, where the elements I, Q, U, and V signify the total light intensity, difference between intensity of linear horizontal and vertical polarizations, difference between intensity of linear polarizations at $\pm 45 \mathrm{deg}$, and difference between intensity of right and left circular polarizations, respectively. Additionally, the comprehensive set of polarization finger prints of the given sample (e.g., myocardial tissue) is described by the Mueller matrix, M, as described below.

Optical polarimetry consists of illuminating the sample with a known incident Stokes vector $\mathbf{S}_{\text {in }}$ and measuring the changes in the output Stokes vector $\mathbf{S}_{\text {out }}$; these changes correspond to the light interactions with the sample, as represented by M. Mathematically,

$\mathbf{S}_{\mathrm{out}}=\mathbf{M} \cdot \mathbf{S}_{\mathrm{in}}$.

The Mueller matrix, M, contains information about all simultaneously occurring polarimetric processes and is measured experimentally. That said, polar decomposition is the most exploited technique to date for decoupling of $\mathbf{M}$ and providing information about individual polarimetry features, as described in detail elsewhere. ${ }^{23}$ Out of these, the significantly dominant metrics in tissue polarimetry (e.g., myocardium) include depolarization and linear retardance, which primarily relies on and provides information about the highly scattering and anisotropic nature of the myocardial tissue, respectively.

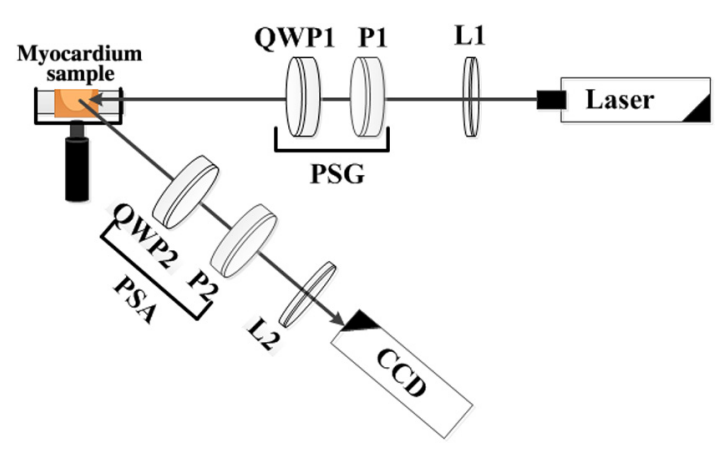

Fig. 1 Schematic of experimental setup for optical polarimetry imaging. The light from the laser source passes through the PSG, illuminates the tissue sample, and is analyzed by the PSA before being detected by the CCD camera. PSG, polarization state generator; PSA, polarization state analyzer; P1, P2, polarizers; QWP1, QWP2, quarter-wave plates; L1, L2, lenses.

The typical imaging setup used for optical polarimetry measurements is shown in Fig. 1. Briefly, the sample (i.e., myocardial tissue) is sequentially illuminated by different input Stokes vectors, generated by passing the light from the source (i.e., laser) through a polarization state generator (PSG), comprising of a linear polarizer P1 and quarter-wave plate QWP1. After interaction with the tissue sample, the scattered light is analyzed by a polarization state analyzer (PSA), consisting of a quarter-wave plate QWP2 and a linear polarizer P2, and finally registered by a detector (usually a CCD camera for imaging setup). Each $\mathbf{S}_{\text {in }}$ is analyzed with different $\mathbf{S}_{\text {out }}$ arrangements in the PSA optics. Typically, the set of $\mathbf{S}_{\text {in }}$ and $\mathbf{S}_{\text {out }}$ comprises linear horizontal, linear vertical, linear $+45 \mathrm{deg}$, linear $-45 \mathrm{deg}$, right circular, and left circular. The minimum number of combinations of $\mathbf{S}_{\text {in }}$ and $\mathbf{S}_{\text {out }}$ necessary to calculate the Mueller matrix $\mathbf{M}$ of the tissue sample are 16, as discussed in detail in our previous studies. ${ }^{14,19}$

\section{Polarimetric Characterization of Infarcted Myocardium}

MI, notorious for its silent lethality, is the major trait of heartrelated deaths globally. ${ }^{24} \mathrm{MI}$ is typically linked to an occlusion of coronary artery, which propels to ischemia, followed by necrosis of the affected cardiomyocytes, and thereafter buildup of collagenous scar tissue. Subsequently, remodeling of the myocardium leads to expansion of the infarct, left ventricular dilation, reduction in the thickness of the ventricular wall, and scar formation; overall, these structural alterations influence the normal cardiac function. ${ }^{7}$

The optical polarimetry approach toward the characterization of infarcted myocardial tissue with emphasis on the uncovering of underlying structural features, such as healthy versus scar component, has previously been employed in rat model. ${ }^{16,17}$ Specifically, 1-mm-thick ex vivo myocardium muscle was optically analyzed, both via point measurements and wide field of view imaging [Fig. 2(a)], with subsequent correlation and validation by histopathological evaluation [Fig. 2(b)]. Importantly, Figs. 2(a)-2(d) has been reproduced with permission. The results revealed a large decrease in linear retardance in the infarcted region of the myocardium compared to healthy tissue [Figs. 2(a) and 2(c)], indicating a disruption in the highly ordered tissue microorganization and thereby loss of anisotropy, presumably due to the necrosis of cardiomyocyte fibers and 

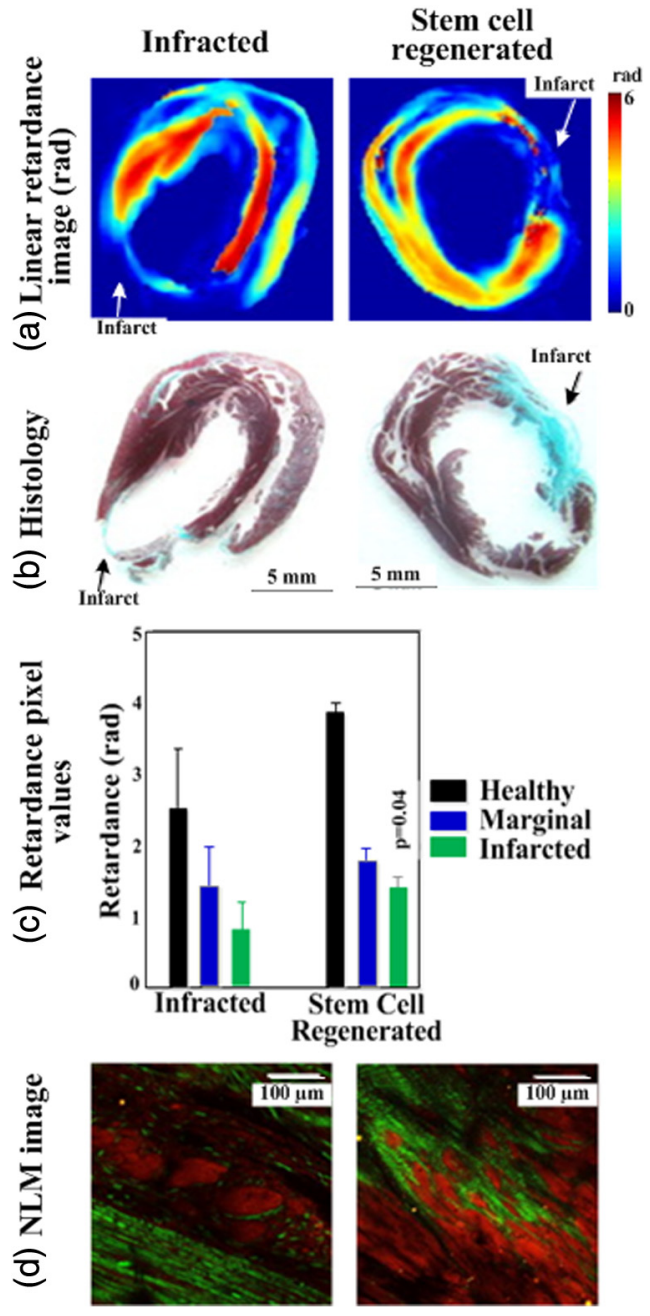

Fig. 2 Illustrative retardance, histology, and NLM images for control infarcted (i.e., no treatment) and stem cell regenerated myocardial tissues. (a) Linear retardance images, (b) corresponding Masson's trichrome images (muscle in red and collagen in blue), (c) mean retardance pixel values for healthy, marginal, and infarcted regions for $n$ (control infarcted) $=4$ and $n$ (stem-cell-treated) $=3$ animals; error bars represent standard error, and (d) representative NLM images from infarcted and stem cell regenerated regions of myocardial samples. The TPEF signal, shown in red pseudocolor arises from cardiomyocytes, and the SHG signal, shown in green pseudocolor arises from collagen. Figure reproduced (from Ref. 16) with kind permission from SPIE (JBO) and Alex Vitkin.

subsequent replacement with scar tissue, where collagen fibers are more randomly oriented, and thus warranting optical isotropy. ${ }^{16,25}$ More specifically, the higher linear retardance (i.e., larger phase shift) observed in healthy myocardium likely arises during propagation of light in the well-aligned myofibers, which are inherently anisotropic (i.e., birefringent) structures. In addition, multiple scattering of light by well-organized myocytes further enhances the linear retardance signal of the tissue. Alternatively, the infarcted myocardium mainly consists of necrotic myocytes where both the structural integrity of individual myocytes and highly ordered microstructure of the tissue is compromised [Fig. 2(b); reproduced with permission], thus reducing linear retardance.

Polarimetry imaging also demonstrated substantial reduction in the thickness of the ventricular wall at the core of infarction, most likely due to necrosis of cardiomyocytes. Moreover, the linear retardance progressively decreased moving from the remote healthy to infarct core through the marginal zone that exhibits somewhat intermediate morphology, i.e., mixed viable and necrotic myocytes. More interestingly, the central region of the myocardial wall revealed distribution of higher linear retardance values, compared to the inner and outer regions (i.e., endoand epimyocardium), perhaps due to the variation in myocytes orientation through ventricular wall, another fascinating application of optical polarimetry in myocardium characterization as discussed in Sec. 6. These optical observations were interpreted and validated by Masson's trichrome histology, where the healthy regions of myocardium were primarily composed of myocytes (stained red) with little collagen (stained blue), contrary to the infarct core region consisting mostly of collagenous scar. Not surprisingly, a marked reduction in the thickness of ventricle at the position of infarct core was also evident in the histology [Fig. 2(b); reproduced with permission]. ${ }^{16}$

Nonlinear microscopy (NLM), which has the potential to provide a wealth of insight to the morphological architecture and composition, responsible here for the measured trends of linear retardance, taken together with the histology findings, offers an opportunity to better recapitulate the results. Specifically, the validation was further extended to more elaborated and specific analyses of collagen fibers and cardiomyocytes in the healthy and infarcted tissues by switching the field of view between second harmonic generation (SHG) and twophoton excitation fluorescence (TPEF), respectively. By means of NLM, the healthy region of the sample expressed highly organized cardiomyocytes with occasional collagen fibers, whereas the opposite trend (i.e., primarily scar collagen and substantially reduced cardiomyocytes) was seen in the infarcted region [Fig. 2(d); reproduced with permission ${ }^{16}$ ], which correlates well and is consistent with the histology findings. Interestingly, NLM also explored special zones of no signals (neither TPEF nor SHG) in the infarct core region, most likely corresponding to regions of necrotic myocytes, not replaced by collagen yet. ${ }^{16,26,27}$

\section{Polarimetric Characterization of Stem-Cell-Regenerated Myocardium}

One innovative biological approach for improving/restoring post-MI cardiac function is the stem cell therapy, where harvesting of the stem cells at the infarcted site may enable regeneration of the myocardial muscle and decline in the collagen content at the scar. ${ }^{9,28}$ On a similar strategy of reconstruction, recent efforts using intramyocardial injection of mesenchymal stem cells into the site of infarction were able to improve cardiac function promisingly. ${ }^{28}$

Engineered remodeling of the myocardial microenvironment at the infarcted site exploiting stem cell therapy has been characterized by optical polarimetry, which illustrated statistically significant rise $(p<0.05)$ in the linear retardance (i.e., anisotropy) at the infarction site [Figs. 2(a) and 2(c); reproduced with permission], ${ }^{16,17}$ indicative of reduction in collagenous scar and regrowth (differentiation) of cardiomyocytes, consequently boosting cardiac function. Specifically, the repopulation of myocytes and remodeling of the tissue matrix enable to strengthen the polarimetric signature (i.e., linear retardance) of the regenerated myocardial tissue. Nevertheless, the recuperating of linear retardance values remains below their healthy counterparts, manifesting the deficiency in complete recovery of cardiac microstructure and function, which is consistent with the studies 
reporting myocardial regenerative treatments that illustrated only partial functional recovery. ${ }^{28}$ Conventional histology and NLM have been used to link these trends in the linear retardance signals with underlying changes in the myofiber architecture and aid in the interpretation [Figs. 2(b) and 2(d); reproduced with permission]. Specifically, histology revealed that the cardiomyocytes from the healthy region have infiltrated into the scar collagen compartment, most likely due to the stimulated regrowth of cardiac muscle from the injected stem cells, as supported by NLM, which ascertained that the repopulation of cardiomyocytes earned the border shrinkage of the collagenous scar compartment after stem cell therapy. Interestingly, a distinct feature of the regrown myocytes, by means of NLM, was the local reorganization of collagen fibers; the alignment in a coherent manner parallel to the myocytes, indicating the unique capability of myocytes to profoundly influence the microstructural properties of the collagen scar at multiple scales. ${ }^{16,26,27,29,30}$ Overall, these remodeling's of the myocardium were reflected in the increasing trend of anisotropy (and linear retardance) toward healthy counterparts, demonstrating the capability of optical polarimetry to visualize the extent and tracking such regenerative treatments of the myocardium.

\section{Polarimetric Characterization of Radiofrequency Ablated Myocardium}

Remodeling of the myocardium after MI, either engineered through stem cell therapy or otherwise, has been speculated to impair the normal electric signaling system of the heart and thereby pose the risk of arrhythmia development. ${ }^{31,32}$ In particular, the action potential properties pertaining to the myofibers at the infarct region frequently differ from normal myocardium and may result in adverse electrical disruption. One effective treatment strategy for focal arrhythmias is electrical ablation, ${ }^{10}$ where selected radiofrequency (RF) energy is deposited at specific spots in the myocardium to isolate the arrhythmogenic circuits. The ablated lesion typically comprises a lesion necrotic core (i.e., the central zone with coagulative necrosis) surrounded by a rim region of intermixed viable and nonviable cells. ${ }^{11,12}$ The characterization of RFA lesion extent within the targeted boundaries is crucial toward assessment of the RFA treatment success.

Optical polarimetry has been used for quantitative assessment of RFA lesions created in myocardial tissue, where depolarization and linear retardance images demonstrated significant visibility of the spatial extent of RF-induced thermal damage. ${ }^{13,14}$ Importantly, polarimetry has earned a reputation by enabling the visualization of clinically important rim region, characterization of which usually presents a major challenge to the conventional medical imaging tools such as US and CT and other large pool of optical imaging techniques. Specifically, these imaging modalities have only focused to distinguish the RFA lesion core from the surrounding healthy myocardium and have not investigated the sandwiched rim region, despite its potential clinical importance, as the residual viable myocytes in this region might complicate or resist the targeted "electric isolation," resulting in RFA treatment failure. Alternatively, quantitative determination of the polarization metrics in the healthy, RFA lesion core, and rim regions illustrated significant differences in the depolarization $(p<0.01)$ and linear retardance $(p<0.05)$ maps [Figs. 3(c) and 3(d); reproduced with permission]. ${ }^{14}$ Mean values for the depolarization and linear retardance magnitude, as calculated from a region of interest
$(\mathrm{ROI})=50 \times 50$ pixels over all samples $[n(\mathrm{RFA})=6, n$ $($ control $)=5$ ], has been shown in Figs. 3(e) and 3(f) (reproduced with permission), respectively; statistical significance in the difference of these polarimetric quantities in the three zones has been calculated with two-tail unpaired $t$-test and shown with arrow annotation here.

The observed contrast in depolarization can be interpreted by considering two factors: multiple scattering and tissue anisotropy. ${ }^{14}$ Multiple scattering of light is primarily due to the complex patterns of refractive index variations of myocardium. Interestingly, thermal coagulation caused by RF currents significantly alters normal tissue architecture, enabling increased scattering; this alone would suggest increased depolarization in RFA lesions, ${ }^{33}$ contrary to the observed depolarization reduction. In addition to multiple scattering, intrinsic anisotropy of the tissue also contributes toward increased depolarization. ${ }^{34}$ Specifically, the linear retardance magnitude and orientation may be spatially inhomogeneous, changing in various tissue microdomains. ${ }^{35}$ Polarized light that passes through these microdomains of inhomogeneous anisotropy undergoes additional randomization, and depolarization increases. Overall, it has been postulated that thermal coagulation caused by RF ablation results in loss of tissue anisotropy, and a corresponding homogenization of the tissue morphology, enabling better preservation of incident light polarization, thus reduced depolarization. ${ }^{14}$

The corresponding histology revealed that the matrix of healthy myocardium was composed of intact and aligned arrays of cardiomyocytes, in contrast to the RFA core region which suffered thermal insult resulting in coagulative necrosis [Fig. 4(a)]. Specifically, Masson's trichrome histology of the RFA core region showed a dark-reddish hue with black zones, structural alteration in cellular membrane and morphology, and compromised nuclear integrity, all markers of extensive thermal damage. ${ }^{11}$ In the context of RF ablation, the polarimetry patterns followed the microstructural changes seen in histology.

By extending the polarimetry analysis of the RFA lesion, objective segmentation of the depolarization image into three (RFA core, rim, and healthy) zones was obtained, with the use of a local fuzzy thresholding algorithm, followed by quantitative comparison with the ground truth segmentation from manual delineation of RFA core and rim regions on the corresponding histology image [Figs. 4(a) and 4(b)]. Qualitative comparison of the two segmentation schemes has been evaluated by the overlap of the corresponding results [Fig. 4(c)], which demonstrated a good qualitative agreement. The comparison of normalized areas (i.e., the ratio of the given segmentcore or rim - area to total area of the sample) of the RFA core and rim regions for the ground truth and computer-aided segmentations also illustrated good agreement [Fig. 4(d)]. Other quantitative evaluation indices for the comparison of the two segmentation schemes were: Dice similarity coefficient $(0.78 \pm 0.02$ and $0.80 \pm 0.02)$, specificity $\left(S_{p}=0.76 \pm 0.17\right.$ and $0.72 \pm 0.17)$, sensitivity $\left(S_{n}=0.83 \pm 0.10\right.$ and $\left.0.91 \pm 0.08\right)$, and accuracy (Acc $=0.81 \pm 0.09$ and $0.71 \pm 0.10$ ) for the RFA core and rim regions, respectively. ${ }^{13}$ Despite the technological advances in the available pool of imaging tools, such quantitative analysis of RFA lesion in three zones remains elusive. The computer-assisted segmentation of parametric depolarization images suggests another application of optical polarimetry, namely its extended use in objective RFA image quantification. 
(a) White light photo

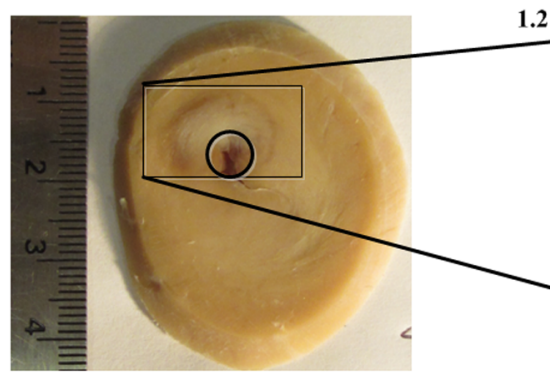

(c) Depolarization $\Delta_{T}(\%)$

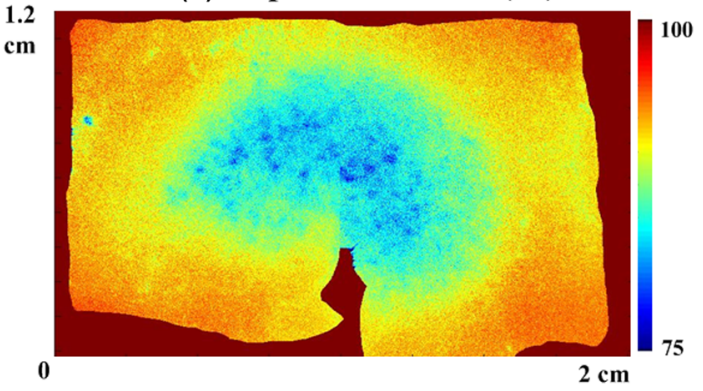

(e) Mean depolarization

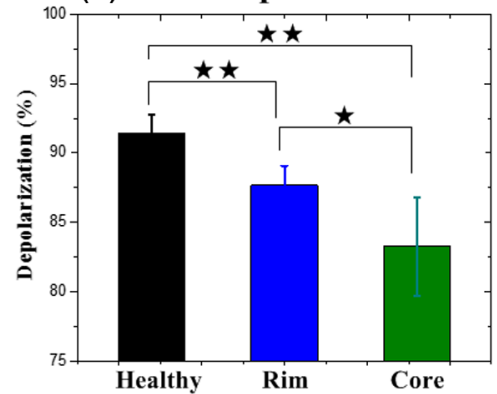

(b) ROI from (a)

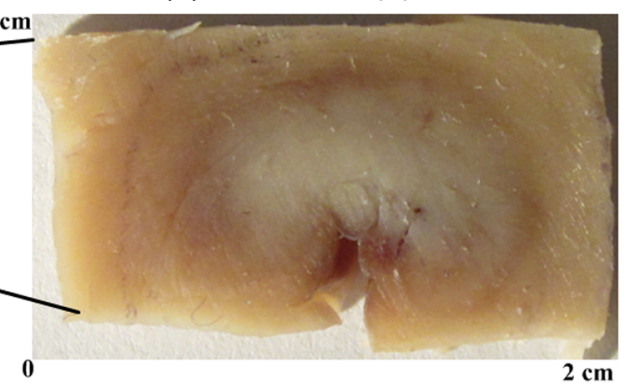

(d) Linear retardance magnitude $\delta(\mathbf{d e g})$

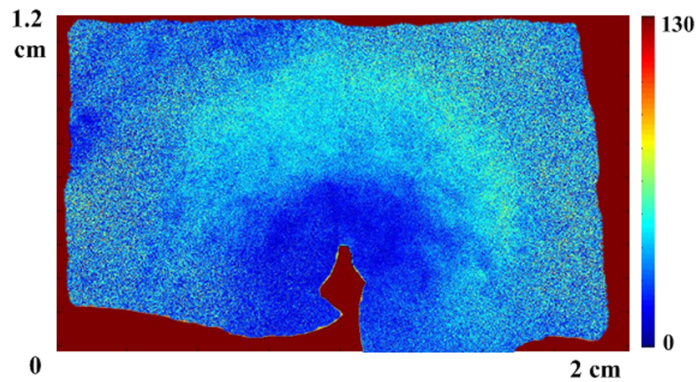

(f) Mean linear retardance magnitude

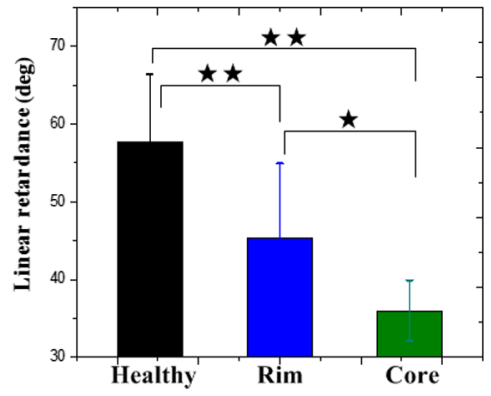

Fig. 3 (a) White light photograph of gross myocardial tissue with RFA lesion (sample from apex), (b) magnified view of region analyzed with optical polarimetry (i.e., $\mathrm{ROI}$ ) from the gross sample, (c) depolarization image, (d) linear retardance magnitude image, (e) mean depolarization, and (f) mean linear retardance magnitude vales for healthy, rim, and core regions of the RFA lesion. $\star=p$ value $<0.05$; $\star \star<0.01$, calculated with two-tail unpaired $t$-test; Error bars show standard error. $n(\mathrm{RFA})=6, n($ control $)=5$. Figure reproduced from Ref. 14 with permission.

\section{Polarimetric Characterization of Cardiomyocytes Orientation}

Myofiber organization in cardiac muscle is crucial not only toward heart contractile function but also to the conduction of electric potential. In general, the orientation of the myofiber undergoes a 180-deg rotation from the epi- to endocardium of the ventricle. ${ }^{36}$ In addition to conventional histology image analysis, ${ }^{37}$ diffusion-tensor MRI, ${ }^{38}$ US elastic tensor imaging, ${ }^{39}$ optical coherence tomography, ${ }^{40}$ etc. have been intensively investigated for assessment of the myofiber organization in cardiac muscle.

The myocytes being fibrous structures, as mentioned earlier, are associated with optical anisotropy (birefringence), which can be sensitively characterized with optical polarimetry. Specifically, the direction of linear retardance, an important polarization metric, has been readily correlated with the underlying orientation of cardiomyocytes, as explored in many optical studies. For instance, the organization of myocytes at the septum and apex of myocardium has been determined with optical polarimetry, with subsequent validation from histology. ${ }^{14}$
Keeping in view the overall complex orientation pattern of myocytes, the geometrical alignment of the myocardial tissue with respect to the probing beam is expected to significantly influence the measured linear retardance, both magnitude and direction, which may subsequently lead to an ambiguity in the orientation of myofibers as assessed by polarimetry. However, acknowledging the importance of and aiming to sketch the intrinsic orientation of myocytes, polarimetrybased geometry-independent methods have been developed. ${ }^{15,22}$ Briefly, a dual-projection polarimetry methodology has been proposed and implemented, whereby the myocardial sample is imaged twice at different incident beam angles, generating sufficient information to reconstruct the intrinsic orientation of the linear retardance and thereby the myocytes. The dual-projection method was tested in both healthy and infarcted cardiac models whereby the results provided a more intrinsic indicator of the tissue anisotropy axis and better insight into tissue structure. A comparative study showed that the dual-projection polarimetry technique yields reasonably good agreement with diffusion tensor MRI, an extensively used imaging tool for probing the anisotropy axis based on tissue water diffusivity. ${ }^{15}$ 
(a) Masson's trichrome histology

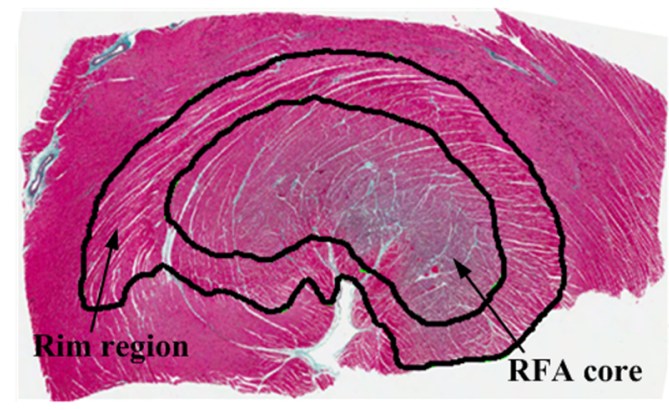

(c) Overlap from (a) and (b)

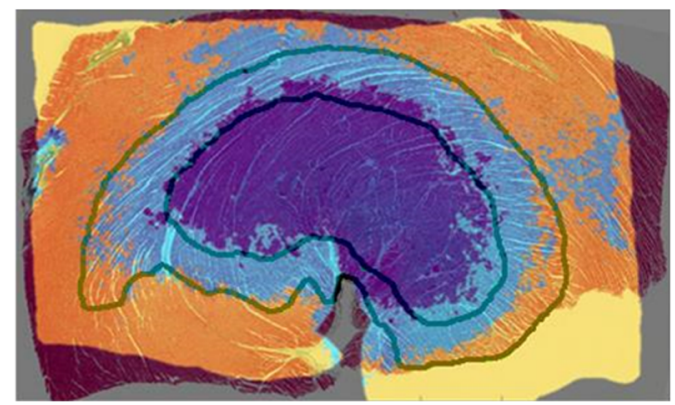

(b) Computer-aided segmentation

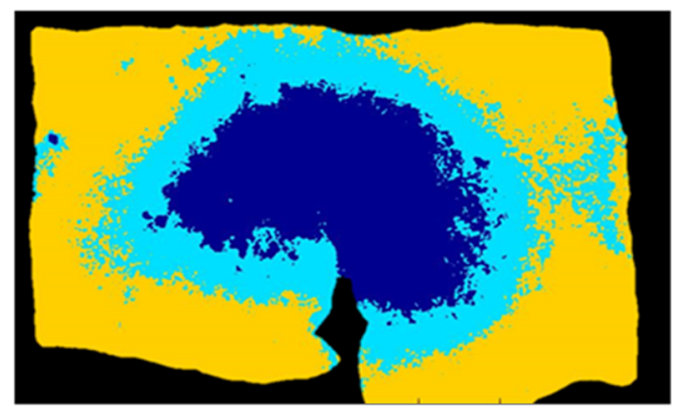

(d) Segmented normalized areas

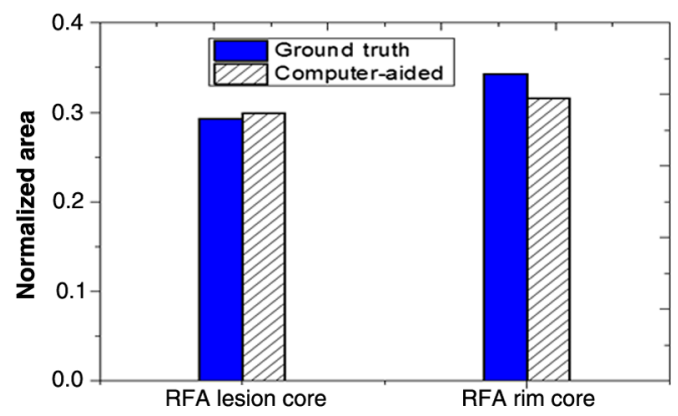

Fig. 4 (a) Ground truth segmented histology image; RFA core and rim regions are demarcated by the black contours, (b) computer-aided segmentation using local fuzzy thresholding algorithm; RFA core, rim and healthy regions are represented by pseudocolors dark blue, light blue, and yellow, respectively. Black represents the background, (c) overlapped images from (a) and (b) illustrating good qualitative agreement of the ground truth and computer-aided segmentation schemes, and (d) comparison of normalized area ratios (with respect to total sample area) for RFA lesion core and rim region segments as calculated using ground truth and computer-aided algorithms.

Collectively, these studies indicate that optical polarimetry may also prove useful in exploring the myofibers organization in pathologies involving dysfunction of the myocardium, where the ventricular remodeling alters muscle fiber alignment.

\section{Polarimetric Characterization of Myocardium in Clinics: Opportunities and Limitations}

Optical polarimetry is a rapidly growing imaging method in biomedical research and has witnessed significant advancements in the recent years. Improvements in the basic characteristics (e.g., robustness, speed, accuracy, resolution, etc.) of a polarimetry imaging system are primarily linked to the polarization modulating components in both the PSG and PSA arms. For instance, photoelastic modulators and liquid crystals-based systems, which exploit electronic modulation of polarization states without mechanically moving parts, are significantly fast, capable of polarization imaging in millisecond time scales. ${ }^{41}$ Diffraction components (e.g., polarizing prisms, graded index lenses, gratings, etc.) based systems are the fastest, but they may limit the imaging resolution over a large field of view. ${ }^{42,43}$ In the context of these fast imaging approaches, various proposed polarimetry methods to measure linear retardance, both magnitude and direction simultaneously, in real time, may also help shaping its roots in clinics of cardiac imaging. ${ }^{44,45}$ Integrating polarimetry imaging with other optical modalities such as diffuse reflectance imaging is another convincingly emerging direction to potentially enhance signal strength, particularly for intraoperative tissue characterization. ${ }^{46}$ Likewise, optical polarimetry in tandem with desorption electrospray ionization mass spectrometry imaging enabled 10-fold fast and accurate pathological (i.e., cancer) zone identification in ex vivo tissue slices. ${ }^{47}$ With the above-mentioned innovative advances in polarimetric imaging tools, this procedure may be widely adopted in the future.

Overall, the reported progress of optical polarimetry in characterizing the myocardial tissues demonstrates the integrity of considering this approach to visualize, identify, and quantify myocardial pathologies. However, like other optical imaging modalities, polarimetry has been mostly investigated in preclinical studies, such as for ex vivo myocardium tissue characterization, intraoperative examination of excised tissues, preliminary in vivo studies in dermatology and ophthalmology. ${ }^{20,37,48-50}$ To facilitate the promotion of optical polarimetry in cardiac imaging clinics, the development of a flexible optical probe capable of intracardiac pathology assessment is desirable, which can in times be integrated with, for example, RF ablation catheter for the real time assessment of thermal lesion, similar to the recently reported combined RFA-optical coherence reflectometry catheters. ${ }^{51}$ Indeed, the construction of flexible fiber-based probes for in vivo polarimetric studies is under way, ${ }^{52}$ which, if successfully translated to the clinics, can possibly contribute in reducing the rate of unsuccessful ventricular arrhythmias treatments $(\sim 10 \%) .{ }^{53,54}$ Assessment of other cardiac pathologies such as the MI lesion (spatial extent, geometry, and position) and tacking the progress of stem cell therapy can also be made possible through the proposed optical probe, most likely delivered to the myocardial wall via catheter. 
In vivo optical measurements (including polarimetry) for the assessment of myocardial pathologies such as infarcted tissue and RFA lesions are always vulnerable to the presence of blood in the tissue. The optical signal arising from the blood would inevitably contaminate the signal from the given myocardial pathology. Specifically, the presence of blood both moving within the tissue and leaked by a surgical procedure would inevitably alter the polarimetric signatures from the myocardial tissues. Importantly, the interaction of polarized light with the blood would mainly cause depolarization. ${ }^{55}$ Specifically, the blood is a fluid tissue that is composed of various diluted scatterers (i.e., erythrocytes, leukocytes, and platelets) having different shapes (i.e., disk-like biconcave, spherical, and biconvex) and sizes (i.e., from $\sim 2 \mathrm{~mm}$ diameter for platelets to $\sim 18 \mathrm{~mm}$ diameter for leukocytes). ${ }^{56,57}$ These scatterers float freely and are generally separated from one another by plasma. However, the presence of any birefringent (i.e., fibrous) structure in the blood seems scarce. Consequently, the major polarimetric signal arising from the blood present in the myocardial tissue would be depolarization, and minimal/no signal of linear retardance. ${ }^{55}$ In this context, it is postulated that the contribution of linear retardance signal arising from the blood would be insignificant.

Optical polarimetry images are limited to depth-weighted composite analysis of tissue polarimetric signatures, and are not depth resolved. Specifically, the average sampling depth of optical polarization for biological tissues, e.g., myocardium, in the visible spectrum is 4 to $6 \mathrm{~mm}$, as reported by both simulation and experimental studies. ${ }^{58,59}$ That said, the polarimetry images would represent depth-weighted averages over such thicknesses. Nevertheless, this issue did not limit the ability to visualize and evaluate the polarimetric differences in the healthy and pathological myocardium substrate, as often revealed by the comparison with histopathology.

The power that most likely drives the development of optical polarimetry is that it visualizes biological samples such as myocardium with high-resolution nondestructively, meaning that it offers an attractive candidate for noninvasive characterization of myocardial pathologies: while the so far reported studies mostly used fixed and sectioned samples, realization of similar in vivo applications (without damaging tissues) has already begun. ${ }^{60,61}$ This also underscores the potential of optical polarimetry to provide complementary information derived from histology or microscopy, particularly when tissue sectioning is an option.

\section{Conclusion}

In this review, the use of optical polarimetry for characterization of myocardial tissues has been discussed, particularly in the context of tissue imaging. These preclinical studies using polarization-gated imaging (i.e., optical anisotropy and depolarization monitoring) illustrate the advantages of optical polarimetry in probing the ultrastructure of myocardial tissues exhibiting typical pathologies such as infarction, stem cell regeneration, and RFA ablation. Specifically, optical polarimetry of myocardial tissues may thus prove useful in evaluating the effects of disease progression (e.g., infarction) and treatment monitoring (e.g., stem cell therapy and RF ablation), thereby providing a functional feedback to the treatment efficacy. Although these preliminary applications have gotten their feet in the door, the clinical realization still faces significant challenges.

\section{Disclosures}

The author declares no conflict of interest.

\section{Acknowledgments}

Permission granted by SPIE (JBO) for the reuse of material from Ref. 16 of this paper is highly appreciated. The author is also thankful to Dr. Rida Safdar for her valuable inputs during the manuscript preparation.

\section{References}

1. B. I. Jugdutt, "The dog model of left ventricular remodeling after myocardial infarction," J. Card. Failure 8(6 Suppl.), S472-S475 (2002).

2. B. I. Jugdutt, M. J. Joljart, and M. I. Khan, "Rate of collagen deposition during healing and ventricular remodeling after myocardial infarction in rat and dog models," Circulation 94, 94-101 (1996).

3. K. T. Weber et al., "Collagen network of the myocardium function, structural remodeling and regulatory mechanisms," J. Mol. Cell. Cardiol. 26(3), 279-292 (1994).

4. Y. Sun and K. T. Weber, "Infarct scar: a dynamic tissue," Cardiovasc. Res. 46(2), 250-256 (2000)

5. L. M. Monteiro et al., "Restoring heart function and electrical integrity: closing the circuit," npj Regener. Med. 2, 9 (2017).

6. G. F. Tomaselli and D. P. Zipes, "What causes sudden death in heart failure?" Circulation 95(8), 754-763 (2004).

7. A. M. Van Der Laan, M. Nahrendorf, and J. J. Piek, "Healing and adverse remodelling after acute myocardial infarction: role of the cellular immune response," Heart 98(18), 1384-1390 (2012).

8. M. Jain et al., "Cell therapy attenuates deleterious ventricular remodeling and improves cardiac performance after myocardial infarction," Circulation 103, 1920-1927 (2001).

9. D. Orlic et al., "Bone marrow cells regenerate infarcted myocardium," Nature 410, 701-705 (2001).

10. O. J. Eick, "Temperature controlled radiofrequency ablation," Indian Pacing Electrophysiol. J. 2(3), 66-73 (2002).

11. S. Thomsen, "Mapping of thermal injury in biologic tissues using quantitative pathologic techniques," Proc. SPIE 3594, 82-95 (1999).

12. A. C. Lardo et al., "Visualization and temporal/spatial characterization of cardiac radiofrequency ablation lesions using magnetic resonance imaging," Circulation 102(6), 698-705 (2000).

13. I. Ahmad et al., "Polarization image segmentation of radiofrequency ablated porcine myocardial tissue," PLoS One 12(4), e0157173 (2017).

14. I. Ahmad et al., "Polarimetric assessment of healthy and radiofrequency ablated porcine myocardial tissue," J. Biophotonics 9(7), 750-759 (2016).

15. M. A. Wallenburg et al., "Comparison of optical polarimetry and diffusion tensor MR imaging for assessing myocardial anisotropy," $J$. Innovative Opt. Health Sci. 03(02), 109-121 (2010).

16. M. F. Wood et al., "Polarization birefringence measurements for characterizing the myocardium, including healthy, infarcted, and stem-cellregenerated tissues," J. Biomed. Opt. 15(4), 047009 (2010).

17. M. F. G. Wood et al., "Polarized light based birefringence measurements for monitoring myocardial regeneration," Proc. SPIE 7179, 717908 (2009).

18. I. Ahmad et al., "Polarimetry based partial least square classification of ex vivo healthy and basal cell carcinoma human skin tissues," Photodiagn. Photodyn. Ther. 14, 134-141 (2016).

19. I. Ahmad et al., "Ex vivo characterization of normal and adenocarcinoma colon samples by Mueller matrix polarimetry," J. Biomed. Opt. 20(5), 056012 (2015).

20. S. Alali et al., "Assessment of local structural disorders of the bladder wall in partial bladder outlet obstruction using polarized light imaging," Biomed. Opt. Express 5(2), 621-629 (2014).

21. N. Ghosh et al., "Mueller matrix decomposition for polarized light assessment of biological tissues," J. Biophotonics 2(3), 145-156 (2009).

22. M. A. Wallenburg et al., "Polarimetry-based method to extract metrics of tissue anisotropy," Opt. Lett. 35(15), 2570-2572 (2010).

23. S. Y. Lu and R. A. Chipman, "Interpretation of Mueller matrices based on polar decomposition," J. Opt. Soc. Am. A 13(5), 1106-1113 (1996). 
24. S. Johansson et al., "Mortality and morbidity trends after the first year in survivors of acute myocardial infarction: a systematic review," $B M C$ Cardiovasc. Disord. 17(1), 53-60 (2017).

25. P. Whittaker et al., "Analysis of healing after myocardial infarction using polarized light microscopy," Am. J. Pathol. 134(4), 879-893 (1989).

26. C. J. Goergen et al., "Microstructural characterization of myocardial infarction with optical coherence tractography and two-photon microscopy," Physiol. Rep. 4(18), e12894 (2016).

27. M. A. Wallenburg et al., "Two-photon microscopy of healthy, infarcted and stem-cell treated regenerating heart," J. Biophotonics 4(5), 297-304 (2011).

28. R. Passier, L. W. Van Laake, and C. L. Mummery, "Stem-cellbased therapy and lessons from the heart," Nature 453, 322-329 (2008).

29. M. A. Wallenburg et al., "Two-photon microscopy of healthy, infarcted and stem-cell treated regenerating heart," J. Biophotonics 4(5), 297-304 (2011).

30. B. C. Wilson, I. A. Vitkin, and D. L. Matthews, "The potential of biophotonic techniques in stem cell tracking and monitoring of tissue regeneration applied to cardiac stem cell therapy," J. Biophotonics 2(11), 669-681 (2009).

31. A. Kléber and Y. Rudy, "Basic mechanisms of cardiac impulse propagation and associated arrhythmias," Physiol. Rev. 84(2), 431-488 (2004).

32. T. A. Quinn, "The importance of non-uniformities in mechano-electric coupling for ventricular arrhythmias," J. Interv. Card. Electrophysiol. 39(1), 25-35 (2014).

33. C. P. Fleming et al., "In vitro characterization of cardiac radiofrequency ablation lesions using optical coherence tomography," Opt. Express 18(3), 3079-3092 (2010).

34. M. Oeff et al., "Effects of multipolar electrode radiofrequency energy delivery on ventricular endocardium," Am. Heart J. 119(3), 599-607 (1990).

35. S. L. Jacques, Biomedical Photonics Handbook, T. Vo-Dinh, Ed., Vol. 2, CRC Press, Boca Raton, Florida (2015), Ch. 12.

36. L. Healy, Y. Jiang, and E. Hsu, "Quantitative comparison of myocardial fiber structure between mice, rabbit, and sheep using diffusion tensor cardiovascular magnetic resonance," J. Cardiovasc. Magn. Reson. 13(1), 74 (2011).

37. D. D. Streeter and D. L. Bassett, "An engineering analysis of myocardial fiber orientation in pig's left ventricle in systole," Anat. Rec. 155(4), 503-511 (1966).

38. D. E. Sosnovik et al., "Diffusion MR tractography of the heart," J. Cardiovasc. Magn. Reson. 11(1), 47-61 (2009).

39. W. Lee, "Ultrasound elastic tensor imaging: comparison with MR diffusion tensor imaging in the myocardium," Phys. Med. Biol. 57(16), 5075-5095 (2012).

40. C. Fan and G. Yao, "Imaging myocardial fiber orientation using polarization sensitive optical coherence tomography," Biomed. Opt. Express 4(3), 460-465 (2013).

41. S. Alali, T. Yang, and I. A. Vitkin, "Rapid time-gated polarimetric Stokes imaging using photoelastic modulators," Opt. Lett. 38(16), 2997-3000 (2013).
42. M. W. Kudenov et al., "Snapshot imaging Mueller matrix polarimeter using polarization gratings," Opt. Lett. 37(8), 1367-1369 (2012).

43. J. Chang et al., "Single-shot spatially modulated Stokes polarimeter based on a GRIN lens," Opt. Lett. 39(9), 2656-2659 (2014).

44. M. Mujat and A. Dogariu, "Real-time measurement of the polarization transfer function," Appl. Opt. 40(1), 34-44 (2001).

45. L. Liu et al., "Simultaneous measurement of small birefringence magnitude and direction in real time," Opt. Lasers Eng. 53, 19-24 (2014).

46. D. J. Cuccia et al., "Modulated imaging: quantitative analysis and tomography of turbid media in the spatial-frequency domain," Opt. Lett. 30(11), 1354-1356 (2005).

47. A. Tata et al., "Wide-field tissue polarimetry allows efficient localized mass spectrometry imaging of biological tissues," Chem. Sci. 7, 21622169 (2016).

48. A. Pierangelo et al., "Multispectral Mueller polarimetric imaging detecting residual cancer and cancer regression after neoadjuvant treatment for colorectal carcinomas," J. Biomed. Opt. 18(4), 046014 (2013).

49. S. Alali et al., "Optical assessment of tissue anisotropy in ex vivo distended rat bladders," J. Biomed. Opt. 17(8), 086010 (2012).

50. P. G. Ellingsen et al., "Mueller matrix three-dimensional directional imaging of collagen fibers," J. Biomed. Opt. 19(1), 026002 (2014).

51. X. Guo, M. F. G. Wood, and A. Vitkin, "Monte Carlo study of pathlength distribution of polarized light in turbid media," Opt. Express 15(3), 1348-1360 (2007).

52. S. Alali, Y. Wang, and I. A. Vitkin, "Detecting axial heterogeneity of birefringence in layered turbid media using polarized light imaging," Biomed. Opt. Express 3(12), 3250-3263 (2012).

53. R. Tung, N. G. Boyle, and K. Shivkumar, "Catheter ablation of ventricular tachycardia," Circulation 122(3), e389-e391 (2010).

54. W. G. Stevenson and U. Tedrow, "Preventing ventricular tachycardia with catheter ablation," Lancet 375, 4-6 (2010).

55. V. Sankaran, J. T. Walsh, and D. J. Maitland, "Comparative study of polarized light propagation in biologic tissues," J. Biomed. Opt. 7(3), 300-306 (2002).

56. J. A. G. Rhodin, Histology a Text and Atlas, Oxford University Press, New York (1974).

57. R. G. Kessel, Basic Medical Histology: the Biology of Cells, Tissues, and Organ, Oxford University Press, New York (1998).

58. X. Guo, M. F. G. Wood, and A. Vitkin, "A Monte Carlo study of penetration depth and sampling volume of polarized light in turbid media," Opt. Commun. 281(3), 380-387 (2008).

59. N. Ghosh and I. A. Vitkin, "Tissue polarimetry: concepts, challenges, applications, and outlook," J. Biomed. Opt. 16(11), 110801 (2011).

60. S. Jacques, J. C. Ramella-Roman, and K. Lee, "Imaging skin pathology with polarized light," J. Biomed. Opt. 7(3), 329-340 (2002).

61. V. M. Turzhitsky et al., "Measuring mucosal blood supply in vivo with a polarization-gating probe," Appl. Opt. 47(32), 6046-6057 (2008).

Iftikhar Ahmad received his MSc and PhD degrees in medical physics from PIEAS, Pakistan, in 2007 and 2016, respectively. He is the recipient of IT and EF postgraduate scholarship and an exchange program scholarship for the University of Toronto. His work focuses on the polarimetric characterization of biological tissues. He has published 14 articles in peer-reviewed journals. 